\title{
The IAU Strategic Plan and its Implementation
}

\author{
George Miley \\ ${ }^{1}$ Leiden Observatory, Leiden, University, The Netherlands; email: miley@strw.leidenuniv.nl \\ ${ }^{2}$ IAU Vice President, Education and Development
}

\begin{abstract}
I shall review the content of the IAU Strategic Plan (SP) to use astronomy as a tool for stimulating development globally during the decade 2010 - 2020. Considerable progress has been made in its implementation since the Plan was ratified at the last General Assembly.
\end{abstract}

\section{Introduction}

The IAU Strategic Plan 2010 - 2020: "Astronomy for Development: Building from IYA 2009" was ratified by the IAU General Assembly in August 2009. It is based on the unique power of astronomy as a tool for furthering human and technological capacity building throughout the world. Astronomy is a fundamental science itself and a gateway to physics, chemistry, biology and mathematics. Astronomy has been an important driver for the development of the most sophisticated technology and provides a link to our deepest cultural roots and origin.

\section{The IAU Strategy}

The Plan ftp://ftp.saao.ac.za/outgoing/kg/astro4dev/stratplan_2012update.pdf is a blueprint for using astronomy as a tool for development. The vision is a global one, namely that eventually all countries should participate at some level in astronomical research and that all children throughout the world will be exposed to knowledge about astronomy and the Universe. The strategy of the SP has several components:

1. A strategic phased integrated approach, including primary, secondary and tertiary education, research and public outreach. This will be based on the potential for astronomy research and education in each country, using objective data augmented by advice from experts in the region.

2. Regional involvement. A bottom-up approach, as pioneered during the International Year of Astronomy (IYA2009), involves regional input, including designation of regional institute nodes to coordinate development efforts throughout their region.

3. Special attention to Sub-Saharan Africa. Because of its relative underdevelopment, sub-Saharan Africa is receiving special attention.

4. Using IYA2009 as a springboard. Several IYA "cornerstones" are being continued and supported (e.g. UNAWE, GTTP) and the network of IYA contacts exploited.

5. Enlarging the number of active volunteers. We are recruiting volunteers from amongst members, doctoral students, postdoctoral trainees, non-member experts on education and outreach and amateur astronomers. Expatriates are particularly important.

6. Initiation of new activities. These include semi-popular lectures on inspirational topics and long-term institute twinning between established astronomy institutes and university departments in less developed countries. Stimulating technological expertise is regarded as crucial in all such programmes.. 


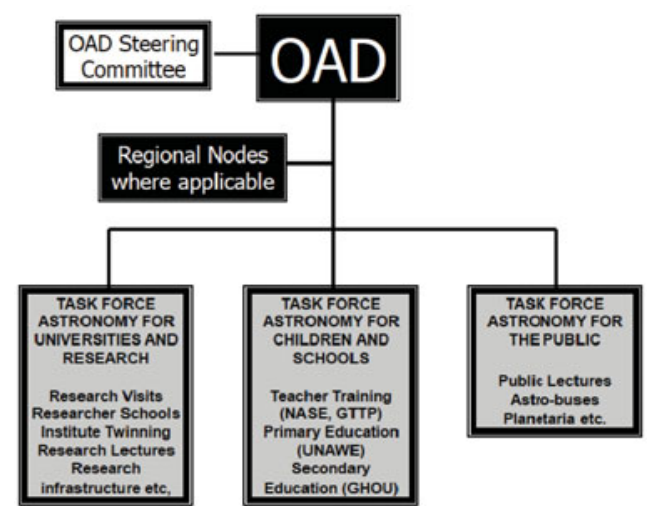

Figure 1. Organisational structure of Astronomy for Development (AfD) activities as envisaged in the SP. Three task forces are being coordinated by the IAU Office of Astronomy for Development (OAD) and regional input ensures a portfolio of demand-driven activities.

7. Exploiting innovative techniques. Innovative approaches to education and development, are being explored, including distance-learning, archives, robotic telescope networks and mobile delivery via astro-buses.

8. Creation of a small global Office of Astronomy for Development (OAD). Mobilising large number of volunteers, implementing new programs and inputting strategic information need professional coordination. Setting up of the IAU OAD has always been regarded as crucial to the success of the SP.

Figure 1 shows the implementation structure as envisaged by the SP. Three Task Forces of experts coordinated by the OAD manage the various AfD activities. The OAD injects a strategic component and safeguards accountability and transparency. Input from the regions ensures that the AfD portfolio matches local needs.

\section{Implementation of the IAU Plan}

The SP covers the ten-year period 20102020 and is being implemented gradually, to match the available funding. The first step was to set up the IAU Office of Astronomy for Development (OAD) to coordinate activities. Following a competitive call for proposals, and the selection of a host organisation by the IAU Executive Committee, the OAD started operation in March 2011. The IAU OAD is a joint venture between the IAU and the South African National Research Foundation (NRF), with Kevin Govender as its first Director. The official launch of the OAD took place on 16th April 2011 by the South African Minister of Science and Technology and the IAU President.

Oversight is provided by a steering committee (Board) with 3 members nominated by both the IAU and the NRF. In considering aspects of the SP most relevant to the IAU, the OAD Steering Committee is enlarged by President and General Secretary of the IAU.

Enormous progress has been made in implementing the IAU Strategic Plan since the last GA. Kevin Govender, the outstanding Director of the OAD, will tell you more about this. Now that the structure envisaged in the Plan is up and running, we are ready to embark on the next phase of its implementation, including fund-raising.

\section{Acknowledgements}

I thank Bob Williams and Ian Corbett for their strong support, the many volunteers in Commissions 46 who pioneered AfD activities over many years, all those involved in the success of IYA 2009, including members of Commission 55 and of course Kevin Govender. 